\title{
BMJ Open Protocol for a placebo-controlled, within-participants crossover trial evaluating the efficacy of intranasal oxytocin to improve pain and function among women with chronic pelvic musculoskeletal pain
}

\author{
Joshua A Rash, ${ }^{1,2}$ Kirsti Toivonen, ${ }^{1}$ Magali Robert, ${ }^{3}$ Maryam Nasr-Esfahani, ${ }^{3}$ \\ John F Jarrell, ${ }^{3}$ Tavis S Campbell ${ }^{1}$
}

To cite: Rash JA,

Toivonen K, Robert M, et al. Protocol for a placebocontrolled, within-participants crossover trial evaluating the efficacy of intranasal oxytocin to improve pain and function among women with chronic pelvic musculoskeletal pain. BMJ Open 2017:7:e014909. doi:10.1136/bmjopen-2016014909

- Prepublication history for this paper is available online. To view these files please visit the journal online (http://dx.doi.org/10.1136/ bmjopen-2016-014909).

Received 26 October 2016 Revised 10 February 2017 Accepted 23 February 2017

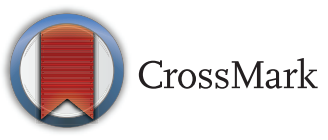

${ }^{1}$ Department of Psychology, University of Calgary, Calgary, Alberta, Canada ${ }^{2}$ Department of Psychology, Memorial University of Newfoundland, St. John's, Newfoundland, Canada ${ }^{3}$ Department of Obstetrics and Gynecology, Cumming School of Medicine, University of Calgary, Calgary, Alberta, Canada

Correspondence to Dr Tavis S Campbell; t.s.campbell@ucalgary.ca

\section{ABSTRACT}

Introduction: This protocol presents the rationale and design for a trial evaluating the efficacy of intranasal oxytocin in improving pain and function among women with chronic pelvic musculoskeletal pain. Oxytocin is a neuropeptide traditionally recognised for involvement in labour, delivery and lactation. Novel evidence suggests that oxytocin decreases pain sensitivity in humans. While oxytocin administration has been reported to lower pain sensitivity among patients experiencing chronic back pain, headache, constipation and colon pain, no research has evaluated the association between intranasal oxytocin and chronic pelvic musculoskeletal pain. The association between oxytocin and pain may differ in women with chronic pelvic musculoskeletal pain relative to other chronic pain conditions because of the abundance of oxytocin receptors in the uterus.

Methods and analysis: This is a prospective, randomised, placebo-controlled, double-blind, withinparticipants crossover trial. 50 women with chronic pelvic musculoskeletal pain will be recruited through a local chronic pain centre and gynaecology clinics. Women will complete baseline measures and be randomised to an experimental or control condition that involve 2 weeks of self-administering twice-daily doses of 24 IU intranasal oxytocin or placebo, respectively. Women will then undergo a 2-week washout period before crossing over to receive the condition that they had not yet received. The primary outcome will be pain and function measured using the Brief Pain Inventory-Short Form. Secondary outcomes include emotional function, sleep disturbance and global impression of change. This trial will provide data on the 14-day safety and side-effect profile of intranasal oxytocin self-administered as an adjuvant treatment for chronic pelvic musculoskeletal pain. Ethics and dissemination: This trial was granted approval from Health Canada and the University of Calgary Conjoint Health Research Ethics Board, and is registered online at ClinicalTrials.gov (\#NCT02888574).

\section{Strengths and limitations of this study}

Methodologically rigorous randomised controlled trial.

- High internal validity, including control over menstrual cycle hormone fluctuations.

- Inclusion criteria may create a sample that does not generalise to all patients with chronic pelvic musculoskeletal pain (eg, results from a sample of women may not generalise to men with chronic pelvic musculoskeletal pain).

Results will be disseminated to healthcare professionals through peer-reviewed publications and to the general public through press releases.

Trial registration number: NCT02888574; Pre-results.

\section{INTRODUCTION}

Oxytocin is produced in the supraoptic and paraventricular nuclei of the hypothalamus. ${ }^{1}$ It is released peripherally into the bloodstream via the posterior pituitary, and into the central nervous system via paraventricular neurons. ${ }^{2}$ While traditionally recognised for involvement in labour, delivery ${ }^{3}$ and lactation, ${ }^{4}$ novel evidence suggests that oxytocin is a safe method for decreasing sensitivity to pain with a low risk of adverse effects. ${ }^{5}{ }^{6} \mathrm{~A}$ systematic review of 38 randomised controlled trials (RCTs) reporting on more than 1500 participants concluded that central administration of 18-40 IU doses of oxytocin produced minimal, non-detectable side effects compared with placebo. ${ }^{7}$

There are three mechanisms through which oxytocin may decrease pain sensitivity. ${ }^{5}$ 
First, a direct hypothalamo-spinal projection originating from the paraventricular nucleus transports oxytocin to the dorsal horn (Lamina-I, II and IV), ${ }^{2}$ an area involved in pain modulation. A subset of neurons in the dorsal horn $(\sim 35 \%)$ contain oxytocin receptors that influence glutamatergic neurons which, in turn, activate GABAergic neurons, resulting in an inhibition of paincarrying A $\delta$-fibres and C-fibres. ${ }^{8-10}$ Second, evidence suggests that oxytocin binds to opioid receptors and may stimulate endogenous opioid release in the brain. An opioid system located in the periaqueductal gray activates a series of descending controls that prevent spinal cord transmission regarding injury. ${ }^{11}$ Oxytocin administered to the periaqueductal gray results in antinociception that can be blocked by the administration of an opioid antagonist. ${ }^{12} 13$ Further, analgesic effects of endogenous and exogenous oxytocin can be blocked by the opioid antagonist naloxone. ${ }^{14}$ The final mechanism involves improving mood, decreasing anxiety and mitigating the stress response. In an informative controlled trial, intranasal administration of oxytocin in men resulted in greater calmness, less anxiety and a trend towards lower cortisol during the Trier Social Stress Test. $^{15}$ Given that negative emotion inductions (eg, anxiety, sadness, anger) are associated with greater pain, ${ }^{16-18}$ along with concomitant heightened autonomic responses, ${ }^{17}$ oxytocin may decrease pain sensitivity by improving mood and anxiety, and buffering the stress response.

Of 33 animal investigations that have assessed the relationship between oxytocin and pain, 29 reported that exogenous oxytocin administration and higher endogenous oxytocin levels decreased pain. ${ }^{5}$ However, an association between oxytocin and pain is less clear in the human literature due to a paucity of methodologically rigorous trials. Thus far, oxytocin administration has been reported to lower pain sensitivity among patients experiencing chronic back pain, ${ }^{19}$ headache,${ }^{20}$ constipation $^{21}$ and colon pain. ${ }^{22}$ It has been difficult to draw firm conclusions about the association between oxytocin and chronic pain in humans from these trials due to methodological limitations, including the lack of an adequate control condition, ${ }^{20}$ use of a delivery method with a high likelihood of confounding pain assessment (eg, intrathecal punch), ${ }^{19}$ peripheral administration ${ }^{22}$ or the recruitment of a sample size that was inadequately powered to detect meaningful effects. ${ }^{21}{ }^{23}$ To date, no research has evaluated the association between intranasal oxytocin and chronic pelvic musculoskeletal pain.

Women report more pain, in more body areas, with greater frequency, and for longer duration than men. ${ }^{24}$ Chronic pelvic pain accounts for 1 in 10 gynaecology outpatient visits ${ }^{25}$ and the prevalence in general practice is similar to that of migraine, back pain and asthma (monthly prevalence of 21.5/1000). ${ }^{26}$ The association between oxytocin and pain may differ in women with chronic pelvic musculoskeletal pain relative to other chronic pain conditions because of a potential peripheral oxytocin-pain pathway. There is an abundance of oxytocin receptors in the uterus, and oxytocin is a potent uterogenic agent that is clinically used in large doses to stimulate uterine contractions and induce labour. ${ }^{2}$ While oxytocin does not cross the blood-brain barrier, the central administration of intranasal oxytocin increases central and blood-plasma oxytocin concentrations. ${ }^{27}$ Thus, intranasal oxytocin administration may be associated with decreased pain through central and peripheral pathways among women with chronic pelvic musculoskeletal pain.

This manuscript details the rationale and design of an RCT evaluating the efficacy of intranasal oxytocin versus placebo in improving pain and function among women with chronic pelvic musculoskeletal pain.

\section{METHODS}

\section{Research questions and objectives}

This research is a pilot study of the efficacy of intranasal oxytocin for improving pain and function among women with chronic pelvic musculoskeletal pain of primarily musculoskeletal origin.

The primary objective is to evaluate the efficacy of intranasal oxytocin for reducing pain.

Secondary objectives are to evaluate the efficacy of intranasal oxytocin for improving mood, emotion function, physical function and sleep.

\section{Study design}

This study is a double-blind, placebo-controlled, withinparticipants crossover RCT adhering to CONSORT guidelines. ${ }^{28}{ }^{29} \mathrm{~A}$ crossover design offers two advantages over a parallel-group RCT: (1) the influence of confounding covariates are reduced because each participant serves as their own control; and (2) statistical power is higher and required sample size to detect meaningful effects is lower. ${ }^{30}$ The trial is registered at ClinicalTrials. gov (\#NCT02888574). Figure 1 depicts a flow diagram of the study design. Participants will be screened for eligibility and, once confirmed, will be randomised to undergo a 2-week experimental or control condition. The experimental and control conditions will involve the twice-daily self-administration of a 24 IU dose of intranasal oxytocin or placebo, respectively. Women will then undergo a 2-week washout period before crossing over to undergo the condition that they did not initially complete. The washout period will ensure that women will be in the same phase of their menstrual cycle (ie, luteal phase) during each nasal spray administration. The primary outcome is self-reported pain. Secondary outcomes include mood, emotion function, physical function and sleep.

\section{Study setting}

Women with chronic pelvic musculoskeletal pain will be recruited from the Calgary Chronic Pain Centre (CPC) 


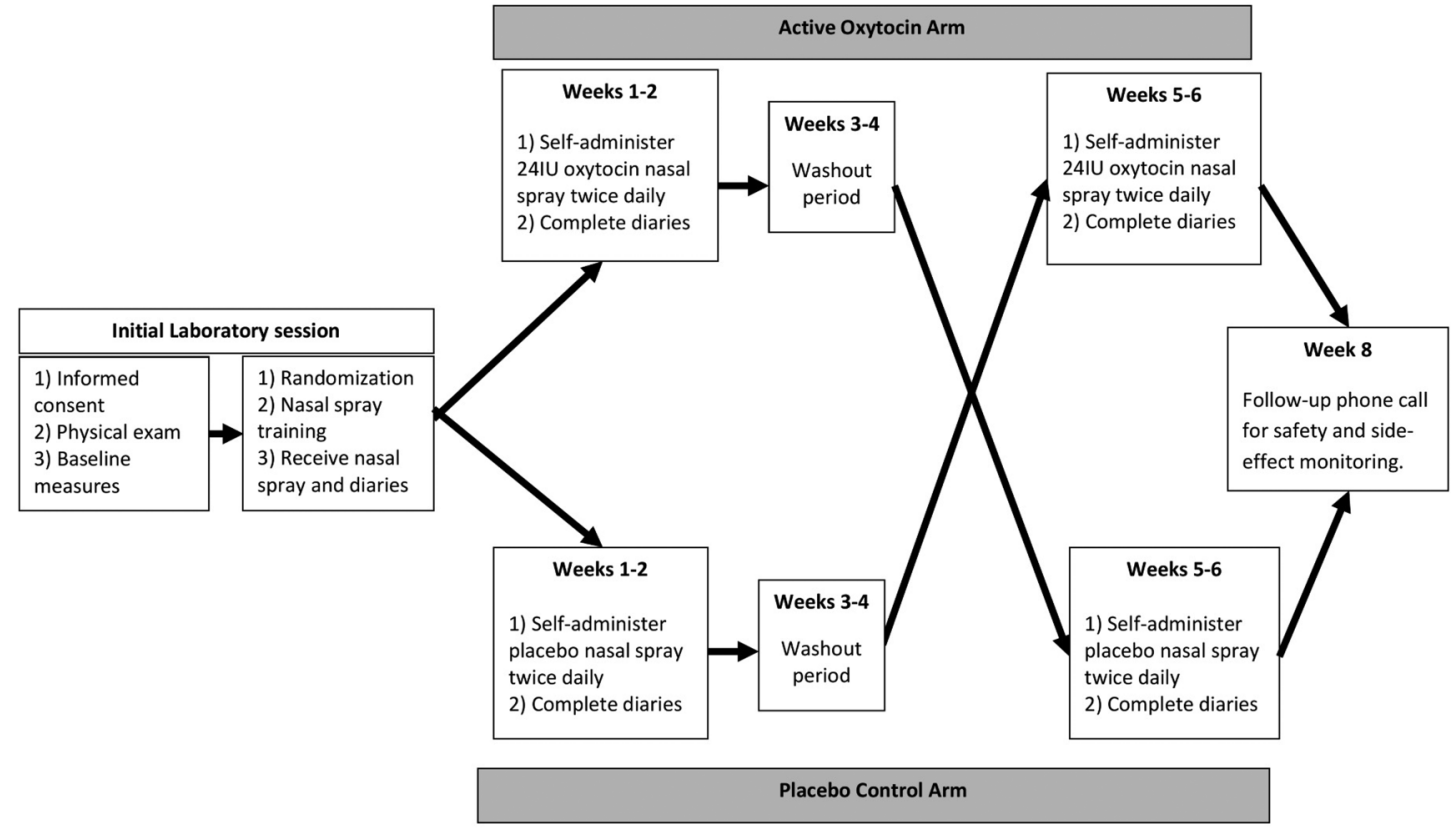

Figure 1 Study flow diagram.

and gynaecology clinics in Calgary, Alberta, Canada. Assessments will occur at the Calgary CPC.

\section{Patient eligibility}

Inclusion criteria

Non-menopausal women with chronic (intermittent or constant) pelvic musculoskeletal pain (ie, pain exceeding 6 months in duration, ie, located primarily in the pelvic region and reproducible on palpation to the muscles spanning the pelvic floor) will be eligible to participate if they: (1) have regular menstrual periods (monthly within a 21-35 day range), given that pain varies across the menstrual cycle ${ }^{31} 32$ and nasal spray administration will occur during the luteal phase; (2) use a permanent, hormonal, or barrier form of contraception, or abstinence in order to minimise the risk of pregnancy during the trial, given that oxytocin is a potent uterogenic agent; (3) can commit to not change their medication during the 6 weeks of this study; and (4) have a moderate amount of pain at baseline (ie, a pain score of 4-7 out of 10 on a numeric rating scale). A baseline pain score of 4-7 out of 10 was selected to prevent floor and ceiling effects and ensure that participants have room to change throughout the course of the study.

\section{Exclusion criteria}

Exclusion criteria include: muscle pain as a result of systemic disease, scoring positive on a urine pregnancy test or contemplating pregnancy, concurrent use of another nasal spray, nasal pathology (eg, ears, nose and throat diagnosis), diabetes insipidus, previous or concurrent use of narcotics delivered intranasally (eg, cocaine) or sacroiliac instability as defined by the European
Guidelines. ${ }^{33}$ Women will also be excluded if they have a primary diagnosis of endometriosis, dysmenorrhoea, interstitial cystitis, functional bowel disorder, fibromyalgia or neuropathic pain. Given that the heart contains oxytocin receptors, ${ }^{34}$ that, when bound to, result in inhibitory effects on cardiovascular activity, ${ }^{35}$ a review of medical chart will be performed and women prescribed antihypertensive medication and those with heart problems (eg, cardiomyopathy, history of myocardial infarction, arrhythmias, prolonged QT interval) will be excluded.

\section{Procedure}

Patient screening, recruitment and enrolment

Fifty women with chronic pelvic musculoskeletal pain will be recruited from the Calgary CPC and directly from the gynaecology clinics of MR and MN-E. Women will be examined by a study gynaecologist (JFJ, MN-E, MR) trained to examine sacroiliac instability and myofascial pain. Chronic pelvic musculoskeletal pain will be confirmed through a physical examination. Any concerns of sacroiliac instability on physical examination will be further assessed by a pelvic physiotherapist. It is estimated that 15 women per month could meet inclusion criteria.

Women will visit the laboratory four times (baseline, 2, 4, 6 weeks). Vital signs (heart rate (HR) and blood pressure (BP)) and self-reported pelvic pain will be monitored at all visits. The first dose of each nasal spray (baseline and 4-week visits) will be supervised and vitals taken every $10 \mathrm{~min}$ for $30 \mathrm{~min}$. Women whose HR and BP decrease by $15 \%$ within this period will be identified as sensitive to the effect of intranasal oxytocin and withdrawn from the trial due to safety concerns. Patient symptoms and side effects will be monitored during the 
2-week, 4-week and 6-week laboratory visits and again over the phone 2 weeks after trial completion.

\section{Baseline assessment}

Testing will occur during the luteal phase of the menstrual cycle (ie, days 14-28) as this is the phase during which women report the highest pain. ${ }^{32}$ At $\sim$ day 14 of their menstrual cycle, participants will attend a baseline session at the Calgary CPC. The objectives of the trial will be explained by a study gynaecologist (JFJ, MN-E, MR) who will also obtain consent to participate. Women will then complete a urine drug and pregnancy test, be randomised to study condition and fill out baseline study questionnaires (refer to table 1 for a schedule of assessments).

\section{Randomisation and blinding}

A research assistant (RA) not involved in study recruitment will use Research Randomizer (http:// www.randomizer.org/) to generate lists of randomly sequenced numbers, stratified by recruiter (MR, MN-E), for assigning patients to condition in a manner consistent with CONSORT. ${ }^{28}{ }^{29}$ Central randomisation will be performed using a 1:1 allocation schedule with random block sizes of 4, 6 and 8 . The allocation sequence will be concealed from the researcher using sequentially numbered, opaque and sealed envelopes. In order to protect against expectation effects and biases, the RA completing baseline assessment and providing participants with their nasal spray will be unaware of condition or nasal spray (oxytocin or placebo), participants will not know what condition they have been assigned to and an RA not affiliated with this study will complete the outcome assessment without being aware of nasal spray that participants are assigned to. Adequacy of blinding will not be formally evaluated, given the lack of evidence for, and concern against conducting such an assessment. ${ }^{29}$

\section{Experimental condition}

During the experimental condition, participants will receive a 2-week course of intranasal oxytocin. Participants will self-administer 24 IU intranasal oxytocin (12 IU delivered to each nostril; Syntocinon, Novartis, Switzerland), two times per day (once in the morning and once in the evening). In order to ensure standardisation of nasal spray administration, participants will be trained in the self-administration of intranasal oxytocin in accordance with published recommendations for the standardisation of oxytocin nasal administration. ${ }^{36}$ Two times per day dosing will ensure elevated central concentration of oxytocin throughout the day, given that salivary concentration of oxytocin was elevated for 7 hours following intranasal administration of $24 \mathrm{IU}$ oxytocin. ${ }^{37}$ Further, a 24 IU dose represents a safe and effective concentration for two times per day dosing (see Rash and Campbell $\left.{ }^{38}\right)$. Oxytocin nasal spray is well tolerated and doses ranging from 10 to 60 IU have produced no reliable side effects in humans relative to placebo. ${ }^{7}$

Table 1 Schedule of assessments

\begin{tabular}{|c|c|c|c|c|c|c|}
\hline \multirow[b]{2}{*}{ Testing variables } & \multirow[b]{2}{*}{ Screening } & \multicolumn{5}{|c|}{ Randomisation } \\
\hline & & Baseline & $\begin{array}{l}\text { Daily } \\
\text { diary }\end{array}$ & $\begin{array}{l}\text { Day } 7 \\
\text { of diary }\end{array}$ & $\begin{array}{l}\text { Day } 14 \\
\text { of diary }\end{array}$ & $\begin{array}{l}\text { Laboratory } \\
\text { follow-ups }\end{array}$ \\
\hline Inclusion and exclusion criteria & $x$ & $X$ & & & & \\
\hline Sociodemographics & & $\mathrm{X}$ & & $x$ & & \\
\hline Medical history & & $\mathrm{X}$ & & $x$ & & \\
\hline Vitals & & $\mathrm{X}$ & & & & $x$ \\
\hline PCS & & $\mathrm{X}$ & & & & \\
\hline MSPSS & & $x$ & & & & \\
\hline \multicolumn{7}{|l|}{ Pain } \\
\hline BPI-SF intensity & & $\mathrm{X}$ & $x$ & & & \\
\hline BPI-SF interference & & $\mathrm{X}$ & $x$ & & & \\
\hline Pelvic pain NRS & $x$ & & & & & $\mathrm{X}$ \\
\hline \multicolumn{7}{|l|}{ Emotional function } \\
\hline PANAS & & $x$ & $\mathrm{X}$ & & & \\
\hline DASS & & $x$ & & $\mathrm{X}$ & $\mathrm{X}$ & \\
\hline \multicolumn{7}{|l|}{ Sleep } \\
\hline MOS-S & & $\mathrm{X}$ & & $\mathrm{X}$ & $\mathrm{X}$ & \\
\hline \multicolumn{7}{|l|}{ Global impression } \\
\hline PGIC & & $\mathrm{X}$ & & & $\mathrm{X}$ & \\
\hline \multicolumn{7}{|l|}{ Expectancy } \\
\hline CEQ & & $X$ & & & & \\
\hline \multicolumn{7}{|l|}{ Side effects } \\
\hline SAS & & & & & $\mathrm{X}$ & \\
\hline
\end{tabular}


Moreover, a recent study of 14 women with fibromyalgia reported that an $80 \mathrm{IU}$ dose of intranasal oxytocin was well tolerated and had no differential side effects from placebo when delivered daily over a 3-week period. ${ }^{23}$ Participants will be instructed to store nasal spray at room temperature (between $15^{\circ} \mathrm{C}$ and $25^{\circ} \mathrm{C}$ ) and outside of range of children.

\section{Control condition}

During the control condition, participants will receive a 2-week course of intranasal placebo (containing the same ingredients as the oxytocin nasal spray except for the active oxytocin) self-administered on the same schedule as the experimental condition. The bottles containing oxytocin and placebo will be identical in appearance, smell, texture and taste. Different coloured stickers will be applied to the bottles in order to distinguish oxytocin from placebo. Only the study coordinator will be aware of what colour signifies which nasal spray and blinding will only be broken should an adverse event occur.

\section{Washout period}

The experimental and control conditions will be separated by a washout period of $\sim 2$ weeks to ensure that intransal administration coincides with the same phase of the menstrual cycle. If women have regular menstrual cycles (by definition $\leq 35$ days, and the luteal phase is the fixed component of the menstrual cycle), the washout phase may be extended by up to 7 days to ensure that all women receive treatment in the same menstrual cycle phase. This time frame will be sufficient, given that the half-life of oxytocin administered centrally using nasal spray is $2-7$ hours and 7 half-lives will be achieved for full clearance in 14-49 hours.

\section{Daily diaries}

Participants will be provided with daily diaries to record their menstrual cycle, time of nasal spray administration, study assessments and side effects (refer to table 1 for a schedule of assessments).

\section{Participant engagement}

A multipronged approach will be used to encourage participant engagement in our trial. First, the timeline and demands of the trial will be explicitly discussed at the outset with participants, who will be asked to sign a behavioural contract to commit to trying to meet the requirements. Second, women will receive telephone reminders prior to each laboratory visit and again if they miss one visit. Third, expectations will be developed for participant attendance. We will ensure that participants know our research staff by name and will be made aware that their research associate has an appointment scheduled with them and will be awaiting their arrival. Fourth, participants who have difficulties attending sessions will be provided with a telephone-based motivational conversation during which ambivalence towards attending sessions will be openly discussed with the goal of securing commitment to attend a session at a time that is convenient for them. Many of these strategies have been identified by Cochrane reviews as methods for improving patient recruitment ${ }^{39}$ and retention. ${ }^{40}$

\section{Measures}

Table 1 shows the measures obtained during each phase of the trial.

\section{Primary outcome}

As recommended by the Initiative on Methods, Measurement, and Pain Assessment in Clinical Trials (IMMPACT) $){ }^{41}$ pain and functional impairment will be measured at baseline and daily using the Brief Pain Inventory-Short Form (BPI-SF ${ }^{42}$ ). The BPI-SF measures pain intensity, the impact of pain on seven daily activities (eg, activity, work, sleep) and analgesic use. The BPI was originally designed to measure cancer pain, but has been shown to be a reliable and valid instrument for measuring non-cancer pain. ${ }^{43-46}$ Test-retest values for pain and interference typically range between 0.72 and 0.98 , and data from studies in many countries have supported a two-factor solution of pain severity and interference. ${ }^{47}$

\section{Secondary outcomes}

As recommended by the IMMPACT, ${ }^{41}$ secondary outcomes include emotional function, sleep disturbance and global impression of change.

Mood will be measured daily using the Positive and Negative Affect Scale $\left(\mathrm{PANAS}^{48}\right)$. The PANAS comprises 10 positive affect words (eg, excited, proud) and 10 negative affect words (eg, distressed, upset). Participants are asked to rate how they feel 'today' using a 5-point Likert scale ranging from 1 'slightly or not at all' to 5 'extremely'. The PANAS has excellent internal consistency (Cronbach $\alpha=0.87-0.90$ ), moderate 8-week testretest reliability $(\mathrm{r}=0.39-0.47)$ and correlates strongly and in the expected directions with measures of mood, well-being, distress and psychopathology. ${ }^{48}$

Emotional function will be measured weekly using the Depression Anxiety Stress Scale (DASS-21 ${ }^{49}$ ). The DASS is a 21-item measure consisting of 3 scales that each comprise 7 items-Depression, Anxiety and Stress. Items refer to the past week and scores range from 0 'did not apply to me at all' to 4 'applied to me very much, or most of the time'. The scales are considered to approximate facets of diagnostic categories, including Depression scale for mood disorders, Anxiety scale for panic disorder and Stress scale for generalised anxiety disorder. ${ }^{50}$ Numerous studies have reported favourable psychometric properties of the DASS in clinical samples $^{50}$ and older patients in primary care. ${ }^{51}$ In patients with chronic pain specifically, the DASS has shown construct validity (ie, was related to other measures of similar constructs) and strong internal consistency (Cronbach $\alpha=0.68-0.96) .{ }^{52} 53$ The DASS has also 
shown moderate to strong 3-month test-retest reliability $(\mathrm{rs}=0.59-0.77)$ in a sample of older adults. ${ }^{54}$

Sleep disturbance will be assessed weekly with the Medical Outcomes Study Sleep Scale (MOS-S ${ }^{55}$ ). The MOS-S is a 12-item self-report measure designed to assess the important dimensions of sleep, including initiation, maintenance, respiratory problems, quantity, perceived adequacy and somnolence. Preliminary support for the MOS-S was provided in the developmental sample of 3445 individuals with chronic illness. ${ }^{56}$ Further support for the reliability and validity of the MOS-S was reported in a nationally representative sample of 1011 US adults and in a sample of 173 adults with neuropathic pain (eg, moderate to strong internal consistency among different subscales; Cronbach $\alpha=0.63-0.83) .{ }^{57}$ Another large study of people with neuropathic pain $(n=603)$ reported a similar Cronbach $\alpha(0.64-0.87)$ as well as good test-retest reliability $(\mathrm{r}=0.67-0.87) .{ }^{58}$

Global change across the course of study will be assessed at the end of each 2-week course of nasal spray using the Patients' Global Impression of Change scale (PGIC $\left.{ }^{59}\right)$. This measure is a single-item rating by participants of their improvement with treatment during a clinical trial on a seven-point scale ranging from 'very much improved', to 'very much worse' with 'no change' as the midpoint. There has been wide use of the PGIC in chronic pain trials, ${ }^{60}{ }^{61}$ and data provide a responsive and readily interpretable measure of participants' assessment of clinical importance of treatment.

\section{Demographics and covariates}

Demographic information will be assessed using a demographic questionnaire, including age, ethnicity, medical comorbidities, medications taken, employment status, marital status, obstetrical history, menstrual history, urinary symptoms, headaches, substance use and smoking status.

Subjective social status is highly correlated with socioeconomic status and will be measured to characterise the sample using the MacArthur Ladder, an image of a ladder meant to represent ranks of social status, on which people mark an ' $\mathrm{x}$ ' on the rung they think best represents their rank. ${ }^{62}$

Concern over pain will be assessed using the Pain Catastrophizing Scale $\left(\mathrm{PCS}^{63}\right)$. The PCS is a 13-item questionnaire that instructs participants to reflect on past painful experiences (eg, 'I worry all the time about whether the pain will end') and indicate the degree to which they experienced each of 13 thoughts or feelings when experiencing pain, on 5-point scales with the anchors of (0) 'not at all' and (4) 'all the time'. The PCS yields a total score and three subscale scores assessing rumination, magnification and helplessness. Internal consistency of the PCS total score is excellent (Cronbach $\alpha=0.93-0.95$ in undergraduate, community and outpatient pain populations) and good for subscale scores (Cronbach $\alpha=0.75-0.95$ ) in undergraduate ${ }^{63} 64$ and outpatient pain samples. ${ }^{65}$ Scores on the PCS are positively correlated $(\mathrm{r}=0.42)$ with a measure of negative thoughts in response to pain ${ }^{64}$ offering support for convergent validity, and have been used to discriminate community and outpatient pain populations. ${ }^{65}$ Finally, total PCS scores have demonstrated adequate test-retest reliability over a mean period of 52 days in a sample of chronic pain patients. ${ }^{66}$

Social support will be measured using the Multidimensional Scale of Perceived Social Support $\left(\right.$ MSPSS $^{67}$ ). The MSPSS is a 12-item scale that measures the perceived availability and adequacy of emotional and instrumental social support offered by family, friends and significant others (eg, 'There is a special person who is around when I am in need'). Participants respond using a 7-point Likert scale ranging between 1 'very strongly disagree' and 7 'very strongly agree'. Empirical evidence suggests that intranasal oxytocin and social support interact to reduce $\operatorname{stress}^{15}$ and social support and oxytocin may also interact to reduce pain. The MSPSS has demonstrated strong internal consistency and test-retest reliability among older adults. ${ }^{68}$

Treatment expectations will be measured using the Credibility/Expectancy Questionnaire $\left(\mathrm{CEQ}^{69}\right)$. The CEQ is a six-item questionnaire that measures two factors-cognitively based credibility of treatment and affective-based expectancy of treatment effectiveness. The scale has a strong internal consistency (Cronbach $\alpha=0.84-0.85$ ) and good test-retest reliability $(\mathrm{r}=0.75-0.82) .{ }^{69}$

Side effects and safety monitoring. As recommended for trials of chronic pain, ${ }^{70}$ side effects will be assessed using open-ended prompts (ie, have you experienced any unwanted symptoms in the past 24 hours) and the Symptom Assessment Schedule $\left(\mathrm{SAS}^{71}\right)$ supplemented with additional symptoms (eg, euphoria, nasal irritation, dizziness) identified in a recent trial evaluating the effect of intranasal oxytocin on pain in a sample of 14 women with fibromyalgia. ${ }^{23}$ Further, all participants will be provided with a number for Health Link (\#811) in order to ensure participant safety. Health Link is a free service in Alberta, Canada, that participants can call 24 hours/day to receive health advice from a registered nurse.

Risk management and discontinuation criteria. Rescue medication is not required with intranasal oxytocin. The best method to resolve side effects is to discontinue use of the drug, which is our recommended course of action. Participants may voluntarily withdraw from the study at any point in time by informing the researcher that they wish to discontinue their participation. Participants are explicitly informed, verbally and in writing, that their decision to discontinue will not impact their future treatment by their physician. Given the limited drug interaction and rare, but non-fatal side effects, we have no a priori defined discontinuation criteria. In accordance with recommendations of the European Medicines Agency, a data monitoring committee is not needed, given that: (1) chronic pelvic musculoskeletal pain is not a life-threatening condition; 
(2) study duration is brief; and (3) intranasal oxytocin has been extensively studied and side effects are rare and benign. ${ }^{7}$

\section{Data analysis}

\section{Sample size calculation}

We powered this study and based our sample size calculation on the number of patients needed to assess our primary outcome: change in self-reported pain between oxytocin and placebo nasal spray. In our previous work, intranasal oxytocin resulted in significant reductions in the report of acute pain (10-15\% reduction in pain), with moderate to large effect sizes $\left(\eta_{\mathrm{p}}^{2}=0.11-0.13\right) .{ }^{72}$ This equated to a minimally clinically significant reduction in pain of $1 \mathrm{~cm}$ on a $10 \mathrm{~cm}$ visual analogue scale. ${ }^{73}$ Given that our previous trial utilised a healthy sample, we powered this trial using a conservative estimate of a medium effect size. Setting power at $90 \%, \alpha=0.05$ and 2-tailed hypothesis testing, 44 patients would need to be recruited to detect a covariate adjusted medium effect $(d=0.50)$. Calculations were performed using GPower. ${ }^{74}$ Fifty patients will be recruited in order to preserve power and account for potential attrition of $15 \%$.

\section{Data management}

Data from this study will be stored and protected in two ways: (1) electronic files will be password-protected and accessed only by the research team; (2) paper files will be stored in a locked filing cabinet within the study site, and will be accessible only to the research team. Data will be deidentified to protect participant confidentiality. An RA blind to study condition will perform data entry and a second RA will randomly sample $25 \%$ of entry values to ensure accuracy. Members of the investigative team will have access to the final trial data set.

\section{Statistical analysis plan}

An intention-to-treat analysis will be performed, ${ }^{75}$ in accordance with CONSORT guidelines, ${ }^{28}{ }^{29}$ to provide an assessment of the practical impact of a treatment. Primary analysis: Improvement in pain following oxytocin nasal spray relative to placebo nasal spray will be assessed using the BPI-SF. Using the linear mixed model function in SPSS, the analytic strategy is a mixed models analysis of covariance with time (baseline, 14 days) as the withinparticipant factor and treatment condition (oxytocin, placebo) as the condition factor after adjusting for relevant covariates. Given that randomisation is expected to eliminate selection bias and equate groups on relevant baseline characteristics, ${ }^{29}$ an a priori decision was made to include only covariates with a strong empirically established association with the dependent variable, rather than include all possible covariates. Any missing data will be handled using multiple imputation in accordance with Harrell's guidelines. ${ }^{76}$ Secondary analyses: The effects of the oxytocin nasal spray on change in mood, emotional function and sleep will be evaluated in a manner analogous to that described above.

\section{Dissemination}

Important protocol modifications will be communicated to the CHREB, Health Canada and study participants. Results of this trial will be communicated through traditional channels (eg, conference presentations and peerreviewed publications). To reach the general public via lay results, press releases detailing study results will be prepared and a study website will be created that details study results.

\section{DISCUSSION}

Data from this study will provide information about the efficacy of intranasal oxytocin to improve pain and function among women with chronic pelvic musculoskeletal pain. Intranasal oxytocin has gained increasing attention in recent years and is showing promising results for pain management, ${ }^{5} 677$ though only a handful of studies have evaluated the effect of intranasal oxytocin on chronic pain in humans. Specifically, intrathecal injection of oxytocin in doses ranging between 50 and $400 \mu \mathrm{g} / \mathrm{kg}$ were reported to reduce pain among adults with acute and chronic low back pain relative to a saline control. ${ }^{19}$ Continuous intravenous administration of oxytocin has led to a dose-response decrease in reports of pain induced by inflating a barostat bag in the descending colon of 26 ( 11 women) patients with abdominal pain. ${ }^{22}$ Relative to placebo, a single intranasal administration of oxytocin was reported to decrease headache in a dose-dependent manner in an otherwise healthy sample of Chinese participants. ${ }^{20}$ The twice-daily administration of intranasal oxytocin over a 13-day period resulted in a significant reduction in abdominal discomfort and a non-significant reduction in abdominal pain in a sample of 49 women with daily abdominal symptoms and chronic constipation, relative to a placebo control. $^{21}$ Post hoc calculation indicated that $60-120$ women would be required to detect an effect of oxytocin on pain. Finally, the daily administration of intranasal oxytocin over a 3-week period did not reduce pain in a sample of 14 women with fibromyalgia relative to a placebo, ${ }^{23}$ though this trial had a number of limitations, including a small heterogeneous sample (refer to Rash and Campbell ${ }^{38}$ for a commentary). This will be the first methodologically rigorous trial to provide data on the efficacy of intranasal oxytocin for use in women with chronic pelvic musculoskeletal pain. To the best of our knowledge, this will also be the first trial assessing the effect of intranasal oxytocin on pain that adequately accounts for important covariates such as stage of menstrual cycle. This is particularly relevant, given that oestrogen has a priming effect on oxytocin synthesis, release and receptor expression. ${ }^{78}$

Despite the recent proliferation of research, few trials evaluating associations between oxytocin and pain have extended from the laboratory into clinical practice where pain is often inadequately managed, ${ }^{79} 80$ and the use of combination drug therapies is advocated as a 
means to improve clinical outcomes and limit deleterious adverse effects by achieving similar therapeutic effects with two compounds, each at a lower dosage. ${ }^{81}$ Oxytocin may be particularly amenable to combination drug therapies due to complimentary mechanisms of actions to opioids and non-steroidal anti-inflammatory drugs, and a low side-effect profile. This trial will provide data on the 14-day safety and side-effect profile of intranasal oxytocin self-administered as an adjuvant treatment for chronic pain.

The proposed trial will speak to the clinical importance of intranasal oxytocin in the treatment of chronic pain. It has been acknowledged that statistically significant results in pain research must be supplemented by consideration of clinical importance of change in a variety of outcomes. ${ }^{73}$ Such information provides a basis for evaluating and comparing the impact of treatments on symptoms, functioning, well-being and overall quality of life. This trial represents the first methodologically rigorous investigation into the efficacy of intranasal oxytocin on chronic pain that adheres to IMMPACT recommendations for core outcome measures for chronic pain clinical trials. ${ }^{41}$ The results of this investigation will speak to the clinical importance of intranasal oxytocin on pain, emotional well-being, physical well-being, mood and sleep. Moreover, the outcome measures selected for use in this trial will allow for direct comparison across clinical trials on women with chronic pelvic musculoskeletal pain.

Finally, an efficacy trial of this nature is a prerequisite before conducting effectiveness research. Intervention trials can be placed on a continuum with highly controlled efficacy trials on one end and 'real-world' effectiveness trials on the other. ${ }^{82}$ Methodologically rigorous RCTs are ideal for efficacy evaluation because such designs minimise bias, enrol a homogeneous patient population, ensure that interventions are delivered in a highly standardised way (including timing and dose) and minimise issues of access, provider recommendation and patient adherence. ${ }^{82}$ For these reasons, an efficacy trial can overestimate an intervention's effects when implemented in clinical practice. Thus, this trial will speak to the efficacy of intranasal oxytocin to manage pain among women with chronic pelvic musculoskeletal pain and support further efficiency trials.

Funding This work is supported by an operating grant awarded by the University of Calgary Clinical Research Fund (project\# 10015623).

\section{Competing interests None declared.}

Ethics approval This study has been approved by the University of Calgary Conjoint Health Research Ethics Board (CHREB) and Health Canada.

Provenance and peer review Not commissioned; externally peer reviewed.

Open Access This is an Open Access article distributed in accordance with the Creative Commons Attribution Non Commercial (CC BY-NC 4.0) license, which permits others to distribute, remix, adapt, build upon this work noncommercially, and license their derivative works on different terms, provided the original work is properly cited and the use is non-commercial. See: http:// creativecommons.org/licenses/by-nc/4.0/

\section{REFERENCES}

1. Viero C, Shibuya I, Kitamura N, et al. Oxytocin: crossing the bridge between basic science and pharmacotherapy. CNS Neurosci Ther 2010;16:e138-56.

2. Gimpl G, Fahrenholz F. The oxytocin receptor system: structure, function, and regulation. Physiol Rev 2001;81:629-83.

3. Fuchs AR, Fuchs F, Husslein P, et al. Oxytocin receptors and human parturition: a dual role for oxytocin in the initiation of labor. Science 1982;215:1396-8.

4. Luhman LA. The effect of intranasal oxytocin on lactation. Obstet Gynecol 1963;21:713-17.

5. Rash JA, Aguirre-Camacho A, Campbell TS. Oxytocin and pain: a systematic review and synthesis of findings. Clin J Pain 2014;30:453-62.

6. Tracy LM, Georgiou-Karistianis N, Gibson SJ, et al. Oxytocin and the modulation of pain experience: implications for chronic pain management. Neurosci Biobehav Rev 2015;55:53-67.

7. MacDonald E, Dadds MR, Brennan JL, et al. A review of safety, side-effects and subjective reactions to intranasal oxytocin in human research. Psychoneuroendocrinology 2011;36:1114-26.

8. Condés-Lara M, Rojas-Piloni G, Martínez-Lorenzana G, et al. Hypothalamospinal oxytocinergic antinociception is mediated by GABAergic and opiate neurons that reduce A-delta and $\mathrm{C}$ fiber primary afferent excitation of spinal cord cells. Brain Res 2009;1247:38-49.

9. Jo YH, Stoeckel ME, Freund-Mercier MJ, et al. Oxytocin modulates glutamatergic synaptic transmission between cultured neonatal spinal cord dorsal horn neurons. J Neurosci 1998;18:2377-86.

10. Breton JD, Veinante P, Uhl-Bronner S, et al. Oxytocin-induced antinociception in the spinal cord is mediated by a subpopulation of glutamatergic neurons in lamina I-II which amplify GABAergic inhibition. Mol Pain 2008;4:19.

11. Melzack R, Wall PD. The challenge of pain. 2nd edn. New York, NY Penguin Books, 1988.

12. Ge Y, Lundeberg T, Yu LC. Blockade effect of mu and kappa opioid antagonists on the anti-nociception induced by intra-periaqueductal grey injection of oxytocin in rats. Brain Res 2002;927:204-7.

13. Yang J, Liang JY, Li P, et al. Oxytocin in the periaqueductal gray participates in pain modulation in the rat by influencing endogenous opiate peptides. Peptides 2011;32:1255-61.

14. Miranda-Cardenas Y, Rojas-Piloni G, Martínez-Lorenzana G, et al. Oxytocin and electrical stimulation of the paraventricular hypothalamic nucleus produce antinociceptive effects that are reversed by an oxytocin antagonist. Pain 2006;122:182-9.

15. Heinrichs M, Baumgartner T, Kirschbaum C, et al. Social support and oxytocin interact to suppress cortisol and subjective responses to psychosocial stress. Biol Psychiatry 2003;54:1389-98.

16. Berna C, Leknes S, Holmes EA, et al. Induction of depressed mood disrupts emotion regulation neurocircuitry and enhances pain unpleasantness. Biol Psychiatry 2010;67:1083-90.

17. Rainville $P$, Bao QV, Chrétien $P$. Pain-related emotions modulate experimental pain perception and autonomic responses. Pain 2005;118:306-18.

18. Rhudy JL, Meagher MW. Fear and anxiety: divergent effects on human pain thresholds. Pain 2000;84:65-75.

19. Yang J. Intrathecal administration of oxytocin induces analgesia in low back pain involving the endogenous opiate peptide system. Spine 1994;19:867-71.

20. Wang YL, Yuan Y, Yang J, et al. The interaction between the oxytocin and pain modulation in headache patients. Neuropeptides 2013;47:93-7.

21. Ohlsson $B$, Truedsson $M$, Bengtsson $M$, et al. Effects of long-term treatment with oxytocin in chronic constipation; a double blind, placebo-controlled pilot trial. Neurogastroenterol Motil 2005;17:697-704.

22. Louvel D, Delvaux M, Felez A, et al. Oxytocin increases thresholds of colonic visceral perception in patients with irritable bowel syndrome. Gut 1996;39:741-7.

23. Mameli S, Pisanu GM, Sardo S, et al. Oxytocin nasal spray in fibromyalgic patients. Rheumatol Int 2014;34:1047-52.

24. Unruh AM. Gender variations in clinical pain experience. Pain 1996;65:123-67.

25. Mathias SD, Kuppermann M, Liberman RF, et al. Chronic pelvic pain: prevalence, health-related quality of life, and economic correlates. Obstet Gynecol 1996;87:321-7.

26. Zondervan K, Barlow DH. Epidemiology of chronic pelvic pain. Baillieres Best Pract Res Clin Obstet Gynaecol 2000;14:403-14.

27. Gossen A, Hahn A, Westphal L, et al. Oxytocin plasma concentrations after single intranasal oxytocin administration-a study in healthy men. Neuropeptides 2012;46:211-15. 
28. Moher D, Hopewell S, Schulz KF, et al. CONSORT 2010 explanation and elaboration: updated guidelines for reporting parallel group randomised trials. Int $J$ Surg 2012;10:28-55.

29. Schulz KF, Altman DG, Moher D. CONSORT 2010 statement: updated guidelines for reporting parallel group randomised trials. PLoS Med 2010;7:e1000251.

30. Senn S. Cross-over trials in clinical research. 2nd edn San Francisco, CA: John Wiley \& Sons, 2002.

31. Pfleeger M, Straneva PA, Fillingim RB, et al. Menstrual cycle, blood pressure and ischemic pain sensitivity in women: a preliminary investigation. Int J Psychophysiol 1997;27:161-6.

32. Riley JL, Robinson ME, Wise EA, et al. A meta-analytic review of pain perception across the menstrual cycle. Pain 1999;81:225-35.

33. Vleeming A, Albert HB, Ostgaard HC, et al. European guidelines for the diagnosis and treatment of pelvic girdle pain. Eur Spine $J$ 2008; 17:794-819.

34. Jankowski M, Hajjar F, Kawas SA, et al. Rat heart: a site of oxytocin production and action. Proc Natl Acad Sci USA 1998;95:14558-63.

35. Petersson M. Cardiovascular effects of oxytocin. Prog Brain Res 2002;139:281-8.

36. Guastella AJ, Hickie IB, McGuinness MM, et al. Recommendations for the standardisation of oxytocin nasal administration and guidelines for its reporting in human research.

Psychoneuroendocrinology 2013;38:612-25.

37. Van IJzendoorn MH, Bhandari R, Van der Veen R, et al. Elevated salivary levels of oxytocin persist more than $7 \mathrm{~h}$ after intranasal administration. Front Neurosci 2012;6:174

38. Rash JA, Campbell TS. Future directions for the investigation of intranasal oxytocin and pain: Comment on: Oxytocin nasal spray in fibromyalgic patients (Rheumatol Int. E-pub ahead of print. doi: 10.1007/s00296-014-2953-y). Rheumatol Int 2014;34:1177.

39. Treweek S, Lockhart $\mathrm{P}$, Pitkethly $\mathrm{M}$, et al. Methods to improve recruitment to randomised controlled trials: Cochrane systematic review and meta-analysis. BMJ Open 2013;3:e002360.

40. Caldwell PH, Hamilton S, Tan A, et al. Strategies for increasing recruitment to randomised controlled trials: systematic review. PLOS Med 2010;7: 1000368.

41. Dworkin RH, Turk DC, Farrar JT, et al. Core outcome measures for chronic pain clinical trials: IMMPACT recommendations. Pain 2005;113:9-19.

42. Cleeland CS, Ryan KM. Pain assessment: global use of the Brief Pain Inventory. Ann Acad Med Singap 1994;23:129-38.

43. Keller S, Bann CM, Dodd SL, et al. Validity of the brief pain inventory for use in documenting the outcomes of patients with noncancer pain. Clin J Pain 2004;20:309-18.

44. Mendoza T, Mayne T, Rublee D, et al. Reliability and validity of a modified Brief Pain Inventory short form in patients with osteoarthritis. Eur J Pain 2006:10:353-61.

45. Tan G, Jensen MP, Thornby Jl, et al. Validation of the Brief Pain Inventory for chronic nonmalignant pain. J Pain 2004:5:133-7.

46. Kapstad H, Rokne B, Stavem K. Psychometric properties of the Brief Pain Inventory among patients with osteoarthritis undergoing total hip replacement surgery. Health Qual Life Outcomes 2010;8:148

47. Cleeland C. The brief pain inventory: user guide. TX: M.D. Anderson Cancer Center, University of Texas, 2009.

48. Watson D, Clark LA, Tellegen A. Development and validation of brief measures of positive and negative affect: the PANAS scales. $J$ Pers Soc Psychol 1988;54:1063.

49. Lovibond P. Manual for the depression anxiety stress scales. Sydney: Sydney Psychology, 1995.

50. Brown TA, Chorpita BF, Korotitsch W, et al. Psychometric properties of the Depression Anxiety Stress Scales (DASS) in clinical samples. Behav Res Ther 1997;35:79-89.

51. Gloster AT, Rhoades HM, Novy D, et al. Psychometric properties of the Depression Anxiety and Stress Scale-21 in older primary care patients. J Affect Disord 2008;110:248-59.

52. Wood BM, Nicholas MK, Blyth F, et al. The utility of the short version of the Depression Anxiety Stress Scales (DASS-21) in elderly patients with persistent pain: does age make a difference? Pain Med 2010;11:1780-90.

53. Sarda J, Jr, Nicholas MK, Pimenta CA, et al. Psychometric properties of the DASS-Depression scale among a Brazilian population with chronic pain. J Psychosom Res 2008;64:25-31.

54. Gomez R, Summers M, Summers A, et al. Depression Anxiety Stress Scales-21: factor structure and test-retest invariance, and temporal stability and uniqueness of latent factors in older adults. J Psychopathol Behav Assess 2014;36:308-17.

55. Hays RD, Stewart A. Ch14: Sleep measures. In: AL Stewart, JE Ware eds. Measuring Functioning and Well Being: The Medical
Outcomes Study Approach. Durham, NC: Duke University Press 1992;235-259.

56. Katz DA, McHorney CA. The relationship between insomnia and health-related quality of life in patients with chronic illness. J Fam Pract 2002;51:229-35.

57. Hays RD, Martin SA, Sesti AM, et al. Psychometric properties of the Medical Outcomes Study Sleep measure. Sleep Med 2005;6:41-4.

58. Rejas J, Ribera MV, Ruiz M, et al. Psychometric properties of the MOS (Medical Outcomes Study) Sleep Scale in patients with neuropathic pain. Eur J Pain 2007;11:329-40.

59. Hurst $\mathrm{H}$, Bolton J. Assessing the clinical significance of change scores recorded on subjective outcome measures. J Manipulative Physiol Ther 2004;27:26-35.

60. Dunkl PR, Taylor AG, McConnell GG, et al. Responsiveness of fibromyalgia clinical trial outcome measures. $J$ Rheumatol 2000;27:2683-91.

61. Farrar JT, Young JP Jr, LaMoreaux L, et al. Clinical importance of changes in chronic pain intensity measured on an 11-point numerical pain rating scale. Pain 2001;94:149-58.

62. Adler NE, Epel ES, Castellazzo G, et al. Relationship of subjective and objective social status with psychological and physiological functioning: preliminary data in healthy, White women. Health Psychol 2000;19:586

63. Sullivan MJ, Bishop SR, Pivik J. The pain catastrophizing scale: development and validation. Psychol Assessment 1995;7:524.

64. Osman A, Barrios FX, Kopper BA, et al. Factor structure, reliability, and validity of the Pain Catastrophizing Scale. J Behav Med 1997;20:589-605.

65. Osman A, Barrios FX, Gutierrez PM, et al. The Pain Catastrophizing Scale: further psychometric evaluation with adult samples. J Behav Med 2000;23:351-65.

66. Lame IE, Peters ML, Kessels AG, et al. Test-retest stability of the Pain Catastrophizing Scale and the Tampa Scale for Kinesiophobia in chronic pain over a longer period of time. J Health Psychol 2008;13:820-6.

67. Zimet GD, Dahlem NW, Zimet SG, et al. The multidimensional scale of perceived social support. J Pers Assess 1988;52:30-41.

68. Stanley MA, Beck GJ, Zebb BJ. Psychometric properties of the MSPSS in older adults. Aging Ment Health 1998;2:186-93.

69. Devilly GJ, Borkovec TD. Psychometric properties of the credibility/ expectancy questionnaire. J Behav Ther Exp Psychiatry 2000;31:73-86

70. Katz NP. The measurement of symptoms and side effects in clinical trials of chronic pain. Contemp Clin Trials 2012;33:903-11.

71. Kristjanson L, Pickstock S, Yuen K, et al. Development and testing of the revised Symptom Assessment Scale (SAS): final report. Perth, WA: Edith Cowan University, 1999.

72. Rash JA, Campbell TS. The effect of intranasal oxytocin administration on acute cold pressor pain: a placebo-controlled, double-blind, within-participants crossover investigation. Psychosom Med 2014;76:422-9.

73. Dworkin $\mathrm{RH}$, Turk DC, Wyrwich KW, et al. Interpreting the clinical importance of treatment outcomes in chronic pain clinical trials: IMMPACT recommendations. J Pain 2008;9:105-21.

74. Faul F, Erdfelder E, Lang AG, et al. G*Power 3: a flexible statistical power analysis program for the social, behavioral, and biomedical sciences. Behav Res Methods 2007:39:175-91.

75. Montori VM, Guyatt GH. Intention-to-treat principle. CMAJ 2001;165:1339-41.

76. Harrell F. Regression modeling strategies: with applications to linear models, logistic and ordinal regression, and survival analysis. New York, NY: Springer, 2001.

77. González-Hernández A, Rojas-Piloni G, Condés-Lara M. Oxytocin and analgesia: future trends. Trends Pharmacol SCi 2014:35:549-51.

78. Light KC, Grewen KM, Amico JA, et al. Oxytocinergic activity is linked to lower blood pressure and vascular resistance during stress in postmenopausal women on estrogen replacement. Horm Behav 2005; $47: 540-8$

79. Benhamou D, Berti M, Brodner G, et al. Postoperative Analgesic THerapy Observational Survey (PATHOS): a practice pattern study in 7 central/southern European countries. Pain 2008;136:134-41.

80. Todd $\mathrm{KH}$, Ducharme J, Choiniere M, et al. Pain in the emergency department: results of the pain and emergency medicine initiative (PEMI) multicenter study. J Pain 2007;8:460-6.

81. Mao J, Gold MS, Backonja MM. Combination drug therapy for chronic pain: a call for more clinical studies. J Pain 2011;12:157-66.

82. Singal AG, Higgins PD, Waljee AK. A primer on effectiveness and efficacy trials. Clin Trans/ Gastroenterol 2014;5:e45. 